\title{
Socioeconomic status and prognosis of heart failure with reduced vs. preserved ejection fraction: A propensity-matched study in the community.
}

\author{
Jose L Puerto ${ }^{1}$, Rocio Gomez ${ }^{2}$, Jose L. Andrey ${ }^{2}$, Sotero P Romero ${ }^{2}$, Maria J. Pedrosa ${ }^{2}$, \\ Manuel Rosety-Rodriguez ${ }^{3}$, and Francisco Gomez ${ }^{2}$ \\ ${ }^{1}$ Hospital Universitario Puerto Real. University of Cadiz, School of Medicine. \\ ${ }^{2}$ Hospital Universitario Puerto Real. University of Cadiz, School of Medicine \\ ${ }^{3}$ Unversity of Cadiz, School of Medicine
}

June 22, 2020

\begin{abstract}
Background: The relationship between socioeconomic status (SES) and the prognosis of HF with reduced (HFrEF) vs. preserved (HFpEF) ejection fraction remains unsettled. Objective: To analyze the relationship between SES and the prognosis of patients with incident HFrEF and HFpEF. Methods: Prospective study over 15 years (2003-2017) on 9658 patients diagnosed with HF. Main outcomes were mortality and hospitalizations for HF. The independent relationship between SES and the prognosis, stratifying patients for cardiovascular co-morbidity after propensity score-matching was analyzed. Results: After matching 7116 patients, during a median follow-up of 8.11 years, 5616 patients died (78.9\%) and 5549 patients were hospitalized (78.0\%). High income level was associated with a lower all-cause mortality (RR for HF patients [95\% CI]: 0.86 [0.80-0.92], RR for HFrEF: 0.88 [0.82-0.95] and RR for HFpEF: 0.82 [0.75-0.90], $\mathrm{P}<0.001$ in all cases), and cardiovascular mortality (RR for HF: 0.84 [0.76-0.92], RR for HFrEF: 0.87 [0.81-0.93] and RR for HFpEF: 0.88 [0.77-0.88], $\mathrm{P}<0.001$ in all cases), less hospitalizations (RR for HF: 0.70 [0.65-0.78], RR for HFrEF: 0.78 [0.68-0.88] and RR for HFpEF: 0.61 [0.55-0.68], P <0.001 in all cases), and less 30-day readmissions (RR for HF: 0.67 [0.59-0.75], RR for HFrEF: 0.71 [0.63-0.79] and RR for HFpEF: 0.61 [0.55-0.69], P $<0.001$ in all cases), after adjustment for comorbidities, and other potential confounders. Analyses of recurrent hospitalizations gave larger SES benefits than time-to-first-event analyses. Conclusions: In this propensity-matched study, a high net annual household income is associated with an improved prognosis of patients with incident HFrEF and HFpEF.
\end{abstract}

\section{INTRODUCTION}

Lower socioeconomic status (SES) is associated with an increased incidence of heart failure (HF) $(1,2)$. Only one study has examined the relationship between SES and incident HF in primary care (3). Mortality (4-6), first hospitalizations $(1,3)$, HF hospitalizations (5-9), and readmissions $(5,6,10)$ are higher in patients with low SES. Only one study has been performed in the community (4), and only one study defines risk separately in men and women (7). Previous studies have not adjusted for covariates like, education, dependency, total wealth, living status, marital status and occupational status, that are closely related to SES and prognosis. SES is a time-varying exposure, but no study so far has considered this in their analyses. Finally and most important, studies including objective measures of cardiac dysfunction have not been performed. Thus, the relationship between SES and the prognosis of HF with reduced (HFrEF) vs. preserved (HFpEF) ejection fraction remains uncertain.

To further analyze the association between SES and the prognosis of HF, HFrEF vs. HFpEF, we put forward the present prospective propensity matched study over 15 years on 9658 patients with incident HF. 


\section{METHODS}

Study design and patients. Prospective study of a cohort of 9658 patients newly diagnosed with HF during a period of 15 years (1 January 2004 to 31 December 2018). These patients are residents of a community of 321,753 inhabitants in the south of Spain, served by the Hospital Universitario Puerto Real (HUPR). The GAMIC cohort comprises adults ( $>=14$ years) diagnosed with HF according to the Framingham criteria (11). All patients have at least one valid echocardiography (EcoC). Patients aged less than 14 years, those not permanently resident in the community of reference and those without a valid EcoC (386 patients, 4.0\%) have been excluded. These 386 patients were mostly women, older and sicker, dying before a valid EcoC could be performed. We have BNP levels in $32 \%$ of the cohort, mainly in patients with obesity and/or chronic pulmonary disease, and only with diagnostic purposes without follow-up determinations. This study has been undertaken with the approval of the Committee for Ethics and Clinical Research, of the HUPR.Collection of data. The data collected provide information on: Sociodemographic and clinical parameters, tests requested, previous treatment, definitive diagnoses, treatment established, scheduled or emergency outpatient visits, and hospitalizations. These data were recorded not only at the time of the inclusion of the patients in the study but also during the 15 years of monitoring. We have recorded data corresponding the 12 months prior to the inclusion of those patients for whom these data were available (data bases of the HUPR and of the Family Doctor). We classified kidney function using the Modification of Diet in Renal Disease equation for estimated glomerular filtration rate (eGFR) based on serum creatinine determinations (12). Comorbidity was assessed by the "modified" Charlson Index (13). The echocardiogram was analyzed following the guidelines of the American Society for Echocardiography (14). The limit for considering the LVEF "normal" has been very variable, between 40 and $50 \%(14,15)$. The criterion that we have chosen to define normal systolic function (LVEF > 50\%) is that customarily utilized in previous studies (15).Socioeconomic status (SES) was self-declared and characterized using net annual household income (NAHIL) measured at inclusion and revised yearly until death or censorship (16). NAHIL was categorized as low ( $<16,000$ euros; $n=3909)$, mid-level $(16,000-30000$ euros ; $n=3377)$, high ( $>30000$ euros; $n=2372)$,or not reported $(\mathrm{n}=35)(16)$. Participants in the last category were not included in our study because of the small sample size (17). As there were no differences of prognosis between patients with HF in the low vs. the NAHIL, the prognostic cut-off for the annual household income level was established using bootstrapping as the point at which the 12 -month probability curve for death exceeds the $97,5^{\text {th }}$ confidence intervals of the same curve for the highest NAHIL. A mean NAHIL of 30,000 euros was associated with a 92-month mortality above the $97.5 \%$ bootstrap levels for the highest NAHIL. Thus, a prognostic cut-off for the NAHIL of 30,000 euros was chosen (18). Educational level was defined as the highest grade or year of school completed, divided into 3 categories, as previously described $(1,2)$.

Using not only income but, the educational level, the marital status, the living status dependency, number of households, occupational status, total wealth and properties value, allows us to more comprehensively capture the cumulative results of SES on cardiovascular health over the life course in participants $(2,16$, 17).

Outcomes. Patients were prospectively included from January 1, 2004, and censored at end of follow-up on December 31, 2018. Primary outcomes included death (all cause and cardiovascular), hospitalizations for HF, and visits for any cause. To confirm mortality and morbidity, the histories of the patients (hospital or health center) were monitored weekly during the period of study. Death was identified from national health service and family practitioners' databases and, deaths that occurred in the emergency department or hospital. When the cause of death was not clear, the physician certifying death was contacted. The patients admitted with heart failure were identified by weekly review of the 9 th revision of the International Classification of Diseases (ICD-9-CM). The codes of the ICD-9-CM included are those previously utilized in other studies: 428, 402.01, 402.11, 425, 429.3, 514, 402.9, 404.01, 404.11, 404.90, 398, 416, and 429 (15). When the health status of any patient was not known, they were contacted by telephone.

Estimation of propensity scores and matching. Because there were significant differences in baseline characteristics among HF patients by income level (Tables 1-3 suppl), we used propensity scores-matching 
to achieve balance (19). We estimated propensity scores (PS) for high income level using a non-parsimonious multivariable logistic regression model (20-22). In that model, all baseline patient characteristics displayed in Tables 1 and 2, and clinically plausible interactions were included as covariates (20-22).

Our PS model discriminated well between patients in the high NAHIL and those in the low/middle NAHIL. The model was fit to data during all steps of the regression analyses (Hosmer and Lemeshow goodness-of-fit test $X^{2}=11.34 ; \mathrm{P}=0.10$, and $c$-statistic $\left.=0.83\right)$. We then used the PS to match each patient in the high NAHIL to another patient in the low/middle NAHIL, who had a similar PS. Thus, matching 2372 of those HF patients in the high NAHIL to another $4744 \mathrm{HF}$ patients in the low/middle NAHIL (Table 4 suppl ). Similarly, 1338 of those patients with HFrEF in the high NAHIL were matched to another 2676 HFrEF patients in the low/middle NAHIL (Table 1 ), and 1034 of those patients with HFpEF in the high NAHIL were matched to another $2068 \mathrm{HFpEF}$ patients in the low/middle NAHIL (Table 2 ). We used a greedy matching algorithm, which first look for matches to five decimal places. The efficacy of PS models is best assessed by estimating post-match absolute standardized differences between baseline covariates. We therefore calculated pre- and post-match absolute standardized differences and presented those findings as Love plots (23). Before matching, the mean PS for HF patients in the low/middle income group was 0.17362 while, in the high income HF group was 0.22186 , in the high income HFrEF group was 0.22218 and, in the high income HFpEF group was 0.22237 , which yielded a standardized difference of $30.7 \%, 29.9 \%$ and $31.0 \%$, respectively, t-test P-value $<0.0001$ in all cases. After matching, the mean PS for HF patients in the low/middle income group was 0.21443 while, in the high income HF group was 0.21449 (standardized difference of $3.0 \%$ and t-test $\mathrm{P}=0.995$ ), in the high income HFrEF group was 0.21449 (standardized difference of $3.1 \%$ and t-test $\mathrm{P}=0.996$ ), and in the high income HFpEF group was 0.21439 (standardized difference of $3.0 \%$ and t-test $\mathrm{P}=0.994$ ).

Analysis of recurrent hospitalizations. Cumulative incidence of hospitalizations and of 30-day readmissions over time were calculated for the NAHIL groups using the Ghosh and Lin non-parametric analysis (24). The rate ratio of HF hospitalizations per 100 patient-years of follow-up (95\%CI and p-value) was calculated based on the Poisson distribution $(25,26)$. We assessed for over-dispersion by consulting the deviance statistic of the Poisson model, and conducted supplementary analyses using negative binomial regression when the deviance statistic exceeded one (26). The negative binomial model was used to modify such an estimated rate ratio by recognizing the heterogeneity (different frailties) of patients with respect to their risks of recurrent hospitalizations (27). Recurrent HF hospitalizations were also analyzed using the Anderson-Gill approach with robust variance estimator that allows for heterogeneity in hospitalization rates between patients (28).

Statistical analysis. All statistical analyses were done with SPSS v 17.0 (SPSS Inc, Chicago, IL). No losses of patients initially included in the study were recorded. We used chi-square tests and independent sample $t$ tests, as appropriate, for descriptive analysis to compare baseline characteristics between pre-match patients by income. For descriptive analysis of post-match cohorts, McNemar tests and paired-sample $t$ tests were used as appropriate. Kaplan-Meier survival analyses and matched Cox proportional hazards models were used to estimate the association between SES and prognosis. We confirm the assumption of proportional hazards by a visual examination of the $\log$ (minus $\log$ ) curves. We conducted formal sensitivity analyses to describe the weight of our evidence, by quantifying the degree of hidden bias that, would need to be present to invalidate our main conclusions (23).

To evaluate the independent risk associated with each of the variables, we utilized multivariate analysis. We identified the most likely predictive variables within each category by backward steps selection, with the variables with probabilistic value $>0.01$ being eliminated from the model. The variables with predictive significance were combined and pre-established covariates were added, in the event of not having been considered in the model. These covariates have been included by virtue of the theoretical likelihood of their association with the prognosis, or by previous studies $(2,7,15)$, or by being considered clinically important for the prediction of morbidity and mortality. In analyzing hospitalizations for heart failure and visits, a sandwich variant estimator was applied in the calculation of the $95 \%$ confidence intervals to account for 
multiple hospitalizations or visits by the same patient $(24,27)$.

Given that income varies overtime, and in order to avoid an optimistic estimation of the results on high income level, performing an analysis with intention to treat, we have considered that patients' NAHIL at inclusion has been the same up to their death, independently of whether NAHIL changed during follow-up. A secondary analysis, incorporated time-varying estimates of high NAHIL and assigned exposure status at the time of an outcome event based on our high NAHIL-exposure algorithm. As comorbidity has been associated with SES, we performed stratified models on patients who, at the time of inclusion, did or did not present comorbidities (Tables 1 and $\mathbf{2}$ ).

Heterogeneity of effects in pre-specified subgroups was examined by testing for treatment-covariate interaction with the Cox proportional hazards regression model, using $\mathrm{p}<.05(29)$.

\section{RESULTS}

Characteristics of the patients. We studied 9658 adults with incident HF, most of them women (5157 patients, 53.4\%), and most of them with HFrEF (5167 patients, 53.5\%) (Table 1 suppl ).

Before matching, there were significant differences between the baseline characteristics of patients in the high income level and those in the low/middle income level (Table 1 suppl ). These differences among the baseline characteristics of HF patients by income were independent of the type of HF, as they were also present in patients with HFrEF (Table 2 suppl) and with HFpEF (Table 3 suppl ). As no difference of mortality between patients with low and middle income was observed, we determined the optimal cut-off point for the net annual household income as 30,000 euros. Therefore, we have performed our analyses by comparing PS matched patients belonging to the highest NAHIL ( $>30,000$ euros) to those belonging to the low/middle NAHIL (up to 30,000 euros).

After matching, all the measured baseline covariates were balanced between HF patients with a high NAHIL vs. those with a low/middle NAHIL (Table 4 suppl), as well as for patients with HFrEF (Table 1 ), and with HFpEF (Table 2 ).

Relationship between the income level and mortality. The number of deaths by income after matching are presented in Table 5 suppl. After matching, during a median follow-up of 8.11 years (interquartile range, $3.37-10.62), 5616$ patients died $(78.9 \%)$ and 4195 patients died of a cardiovascular cause $(59.0 \%)$.

The patients with HF and the highest NAHIL presented a survival longer than that of the patients with a low/middle NAHIL (RR of death for highest NAHIL [95\% CI]: 0.86 [0.80-0.92], P <0.001;Figure 1A ). Similarly, the patients with HFrEF in the highest NAHIL presented a survival longer than that of the HFrEF patients with a low/middle NAHIL (RR of death for highest NAHIL: 0.88 [0.82-0.95], $\mathrm{P}<0.001$; Figure 1B ), and the patients with HFpEF in the highest NAHIL presented a survival longer than that of the HFpEF patients with a low/middle NAHIL (RR of death for highest NAHIL: 0.82 [0.75-0.90], P <0.001; Figure 1C ). The HF patients in the highest income groups showed a cardiovascular mortality significantly lower than that of the patients in the low/middle income groups (RR of death for highest income in HF patients [95\% CI]: 0.84 [0.76-0.98], P <0.001; Figure 1A suppl. RR of death for highest income among HFrEF patients [CI 95\%]: 0.87 [0.81-0.93], P <0.001; Figure 1B suppl. RR of death for highest income among HFpEF patients [95\% CI]: 0.82 [0.77-0.88], $\mathrm{P}<0.001$; Figure $1 \mathrm{C}$ suppl ).

Relationship between the income and the hospitalizations. The hospitalizations and 30-day readmissions are presented in Table $6 \mathrm{suppl}$ and Table $7 \mathrm{suppl}$, respectively. Figure $2 \mathrm{suppl}$ presents the cumulative number of admissions for HF worsening, per 100 patient years (Figure 2A suppl ), and that of 30-day readmission for HF worsening (Figure 2B suppl) in the matched groups. The estimated effects of SES for hospitalization and 30-day readmission for HF by each method considered are presented in Table 8 suppl. Rate ratios for recurrent hospitalizations and 30-day readmissions were lower than those obtained by the proportional-hazards models.

Multivariate relationship between the income and the prognosis.In our primary analysis using an 
intention-to-treat approach, highest NAHIL exposure was associated with a 16\% lower relative risk (RR) of all-cause death, a 15\% lower RR of cardiovascular death, a 17\% lower RR of hospitalization for HF, a 35\% lower RR of hospitalization for a CV cause, a 30\% lower risk of hospitalization for HF, and a $34 \%$ lower RR of 30-day readmission for HF, compared with patients in the low/middle NAHIL, even after adjustment for sociodemographic characteristics, comorbidities, longitudinal use of medications, and propensity to be in the highest income group (Table 3 ). In the second type of analysis, time-dependent exposure to high NAHIL was associated with an even lower adjusted risk of all-cause and cardiovascular death, of hospitalization for $\mathrm{HF}$, and of 30-day readmission for $\mathrm{HF}$, compared with periods with a low/middle NAHIL (Table 9 suppl ). Similarly, high NAHIL was associated with a reduced mortality (all-cause and cardiovascular), hospitalizations and 30-day readmissions in patients with $\mathrm{HFrEF}, \mathrm{HFpEF}$, as well as in men and women with HF (Table 3 and Table 9 suppl ).

This favorable relationship of high NAHIL to mortality was maintained independently that the patients, before their inclusion or during the follow-up, presented cardiovascular events or other comorbidities in Table 1 and Table 2 (highest adjusted hazard ratio [HR] 0.84; 95\% CI: 0.78-0.90, vs. highest HR 0.74; 95\% CI: $0.68-0.80 ; \mathrm{P}<0.01$ in all cases).

Subgroup analyses. These analyses have been performed for patients with HF (Figure $3 \mathrm{~A}$ suppl ), HFrEF (Figure 3B suppl) and with HFpEF (Figure 3C suppl ). Although, age, sex, atrial fibrillation, BMI and comorbidity negatively influenced the effects of income on the prognosis of HF (29), this benefit was also observed in the subgroups of patients over 70 years, women, diabetics, patients with chronic renal disease or with atrial fibrillation, overweight patients and those with an elevated burden of comorbid conditions (Figure 3A-C suppl).

\section{DISCUSSION}

The results of our propensity matched study indicate that in patients with HF, having a NAHIL >30,000 euros is associated with an improved prognosis, decreased mortality and hospitalizations. The most common socioeconomic indicator used in previous studies has been individual (1), household (30), or neighborhood income $(4,10)$. However, income may inadequately reflect socioeconomic position, particularly after retirement. Only a minority of the studies utilizing income addressed this limitation by examining additional socioeconomic indicators such as those employed in our study (30-32).

In comparison to previous observational studies $(1,2-7)$, our study is characterized by its prospective design and comprehensive collection of data on a numerous cohort of patients with incident HF, included in the 15 years study period, with a valid echocardiographic study in all of them. Most of the patients were diagnosed with $\mathrm{HF}$ as outpatients, and they present socio-demographic and clinical characteristics representative of the habitual clinical practice in this type of disease. Both the follow-up over time of the variables and the evaluation of therapeutic compliance have been comprehensive. We set out to perform PS matching and a rigorous statistical analysis of the results, adjusting for covariates not considered by previous studies, that are closely related to SES and prognosis. As SES is a time-varying exposure, we have performed multivariate analyses considering time-dependent exposure to SES. Finally and more important, this is the first study on SES and prognosis of HF including objective measures of cardiac dysfunction, HFrEF vs. HFpEF.

The observed associations between SES and HF prognosis are not fully explained by health-related behaviors, lifestyle factors, or traditional cardiovascular risk factors (32-34). The literature suggests that, the independent associations of SES with cardiovascular outcomes and mortality, at least partially reflect the influence of chronic stressors in daily life, which disproportionately affect persons in low SES groups (35, 36).These chronic stressors might result in maladaptive physiologic coping mechanisms and chronic elevations in blood pressure and inflammation that can lead to physiologic injury of the vasculature and myocardium (8). Once HF is established, deprived groups face numerous challenges, including limited access to healthcare (10), transportation costs, affordability of drug regimens (33), inequalities in treatment (34), greater co-morbidity precipitating cardiac decompensation, fewer contacts with primary care (3), and consequently more reliance on secondary care. Problems are compounded by impaired health literacy, education, and social support 
(35), coupled with poor compliance with medications, diet, and lifestyle restrictions (36). Further, as in our study, education levels correlate with SES (37), patients with higher education may have better understanding and knowledge of their disease process and treatment (38), while patients with lower education levels are less likely to be adherent to therapeutic recommendations (39). In addition, lower education level has been shown to be associated with poor quality of life (40), anxiety (40), physical and emotional distress (41), and inability to actively participate in self-care recommendations (42). Higher levels of education have also been shown to be associated with higher levels of disease-specific knowledge, healthy lifestyle, and improved outcomes (43-45).

Limitations. Firstly, there are limitations derived from an observational study on the habitual clinical practice, which prevent us from discounting completely any residual factors of confusion not determined, and any bias in the selection of the patients, that might explain our results. To reduce the possible influence of these weaknesses in the design of our study, a PS analysis has been performed, adjusting for a wide range of covariates. We have no data on the levels of brain natriuretic peptide (BNP), which constitutes a valuable element for the diagnosis and prognosis of patients with $\operatorname{HF}(46,47)$. In our analysis, we used household income rather than total wealth or education, but adjustment for the educational level, the marital status, the living status, dependency, number of households, occupational status, total wealth and properties value, allows us to more comprehensively capture the cumulative results of SES on cardiovascular health over the life course in participants $(2,16,17)$. We also lack information about the functional status, presence of anxiety or depression, the quality of life of our patients (50), and the literacy of patients or the main caregiver (48). Finally, this is a study carried out in only one center, and in one specific area of the south of Spain, with a population uniformly of white race, with medium-low socioeconomic and educational level, which has universal public health insurance giving people open, cost-free access to the health system (visits, tests and medication), thus limiting generalizability. International geographic variations in event rates has been observed in studies on HF (49).

Acknowledgements: To the members of the Grupo para la atención médica integral y continua de Cádiz (GAMIC), without whose work this paper could not have been written. To Prof. José Almenara for the performance of the statistical analyses and, to Royston Snart for his professional help in the translation of the manuscript into English.

\section{REFERENCES}

1. Shah RU, Winkleby MA, Van Horn L, Phillips LS, Eaton CB, Martin LW, et al. Education, Income, and Incident Heart Failure in Post Menopausal Women: The Women's Health Initiative Hormone Therapy trials. J Am Coll Cardiol 2011;58:1457-1464.

2. Hawkins NM, Jhund PS, McMurray JJV, and Capewell S. Heart failure and socioeconomic status: accumulating evidence of inequality. Eur J Heart Failure 2012;14:138-146.

3. McAlister FA, Murphy NF, Simpson CR, Stewart S, MacIntyre K, Kirkpatrick M, Chalmers J, Redpath A, Capewell S, McMurray JJ. Influence of socioeconomic deprivation on the primary care burden and treatment of patients with a diagnosis of heart failure in general practice in Scotland: population based study. BMJ 2004; 328:1110.

4. Foraker RE, Rose KM, Suchindran CM, Chang PP, McNeill AM, Rosamond WD. Socioeconomic status, med icaid coverage, clinical comorbidity, and rehospitalization or death after an incident heart failure hospitalization: atherosclerosis risk in communities cohort (1987 to 2004). Circ Heart Fail 2011;4:308-316.

5. Bikdeli B, Wayda B, Bao H, Ross JS, Xu X, Chaudhry SI, et al. Place of Residence and Outcomes of Patients with Heart Failure: An Analysis from the Tele-HF Trial. Circ Cardiovasc Qual Outcomes 2014;7:749756.

6. Calvillo-King L, Arnold D, Eubank KJ, Lo M, Yunyongying P, Stieglitz H, Halm EA. Impact of Social Factors on Risk of Readmission or Mortality in Pneumonia and Heart Failure: Systematic Review. J Gen Intern Med 2012;28:269-282. 
7. Jhund PS, MacIntyre K, Simpson CR, Lewsey JD, Stewart S, Redpath A, Chalmers JW, Capewell S, McMurray JJ. Long-term trends in first hospitalization for heart failure and subsequent survival between 1986 and 2003: a population study of 5.1 million people. Circulation 2009;119:515-523.

8. Stewart S, Murphy NF, McMurray JJ, Jhund P, Hart CL, Hole D. Effect of socioeconomic deprivation on the population risk of incident heart failure hospitalisation: an analysis of the Renfrew/Paisley study. Eur J Heart Fail. 2006;8:856-863.

9. Eapen ZJ, McCoy LA, Fonarow GC, Yancy CW, MirandaML, Peterson ED, et al. Utility of Socioeconomic Status in Predicting 30-Day Outcomes After Heart Failure Hospitalization. Circ Heart Fail. 2015;8:473-480.

10. Philbin EF, Dec GW, Jenkins PL, DiSalvo TG. Socioeconomic status as an independent risk factor for hospital readmission for heart failure. American Journal of Cardiology. 2001; 87:1367-1371.

11. McKee PA, Castelli WP, McNamara PM, Kannel WB. The natural history of congestive heart failure: the Framingham study. N Engl J Med 1971; 285: 1441-1446.

12. Rule AD, Larson TS, Bergstralh EJ, Slezak JM, Jacobsen SJ, Cosio FG. Using serum creatinine glomerular filtration rate: accuracy in good health and in chronic kidney disease. Ann Intern Med 2004;141:929-937.

13. Senni M, Santilli G, Parrella P, de Maria R, Alari G, Berzuini C, et al. A novel prognostic index to determine the impact of cardiac conditions and co-morbidities on one-year outcome in patients with heart failure. Am J Cardiol. 2006; 98:1076-82.

14. Vasan RS, Levy D. Defining diastolic heart failure: a call for standardized diagnostic criteria. Circulation 2000;101:2118-2121.

15. Redfield MM, Jacobsen SJ, Burnett JC, Mahoney DW, Bailey KB, Rodeheffer RJ. Burden of systolic and diastolic ventricular dysfunction in the community. Appreciating the scope of the heart failure epidemic. JAMA 2003;289:194-202.

16. Fretz A, Schneider ALC, McEvoy JW, Hoogeveen R, Ballantyne CM, Coresh J, and Selvin E. The Association of Socioeconomic Status With Subclinical Myocardial Damage, Incident Cardiovascular Events, and Mortality in the ARIC Study. Am J Epidemiol. 2016;183:452-461.

17. Diez-Roux AV, Nieto FJ, Tyroler HA, et al. Social inequalities and atherosclerosis. The Atherosclerosis Risk in Communities Study. Am J Epidemiol. 1995;141:960-972.

18. Goode KM, John J, Kilpatrick ES, Bragadeesh T, Clark AL, Cleland JGF. Elevated glycated haemoglobin is a strong predictor of mortality in patients with left ventricular systolic dysfunction who are not receiving treatment for diabetes mellitus. Heart 2009;95:917-923.

19. Sui X, Gheorghiade M, Zannad F, Young JB, Ahmed A. A propensity matched study of the association of education and outcomes in chronic heart failure. Int J Cardiol 2008;129:93-99.).

20. Rosenbaum P, Rubin D. Reducing bias in observational studies using subclassification on the propensity score. J Am Stat Assoc 1984;79:516-524.

21. Rubin D. Using propensity score to help design observational studies: application to the tobacco litigation. Health Services Outcomes Res Methodol 2001;2:169-188.

22. D'Agostino RB Jr. Propensity score methods for bias reduction in the comparison of a treatment to a non-randomized control group. Stat Med 1998;17:2265-2281.

23. Rosenbaum PR. Sensitivity analysis for matching with multiple controls. Biometrika 1988;75:577-581.

24. Ghosh D, Lin DY. Nonparametric analysis of recurrent events and death . Biometrics 2000;56:554-562.

25. Glynn RJ, Buring JE. Ways of measuring rates of recurrent events. BMJ 1996;312:364-367. 
26. Rao JNK, Scott AJ. A simple method for analysing overdispersion in clustered Poisson data. Statistics in Medicine. 1999; 18:1373-1385.

27. Greenwood M, Yule GU. An inquiry into the nature of frequency distributions representative of multiple happenings with particular reference to the occurrence of multiple attacks of disease or of repeated accidents. J Royal Stat Society 1920;83:255-279.

28. Lin DY, Wei LJ. The robust inference for the Cox proportional-hazards model. J Am Stat Assoc 1989;84:1074-1078.

29. Kao DP, Lewsey JD, Anand IS, Massie BM, Zile MR, Carson PE, et al. Characterization of subgroups of heart failure patients with preserved ejection fraction with possible implications for prognosis and treatment response. Eur J Heart Fail. 2015;17:925-935.

30. Christensen S, Mogelvang R, Heitmann M, Prescott E. Level of education and risk of heart failure: a prospective cohort study with echocardiography evaluation. Eur Heart J 2011;32:450-458.

31. Borne Y, Engstrom G, Essen B, Sundquist J, Hedblad B. Country of birth and risk of hospitalization due to heart failure: a Swedish population-based cohort study. Eur J Epidemiol 2011;26:275-283.

32. Williams BR, Zhang Y, Sawyer P, Mujib M, Jones LG, Feller MA, et al. Intrinsic Association of Widowhood With Mortality in Community-Dwelling Older Women and Men: Findings From a Prospective Propensity-Matched Population Study. J Gerontol A Biol Sci Med 2011;66A:1360-1368.

33. Masoudi FA, Baillie CA, Wang Y, Bradford WD, Steiner JF, Havranek EP, Foody JM, Krumholz HM. The complexity and cost of drug regimens of older patients hospitalized with heart failure in the United States, 1998-2001. Arch Intern Med 2005;165:2069-2076.

34. Shah SM, Carey IM, DeWilde S, Richards N, Cook DG. Trends and inequities in beta-blocker prescribing for heart failure. Br J Gen Pract 2008;58:862-869. )

35. Evangelista LS, Rasmusson KD, Laramee AS, Barr J, Ammon SE, Dunbar S, et al.. Health literacy and the patient with heart failure - implications for patient care and research: a consensus statement of the Heart Failure Society of America. J Card Fail 2010;16:9-16.

36. Ghali JK, Kadakia S, Cooper R, Ferlinz J. Precipitating factors leading to decompensation of heart failure. Traits among urban blacks. Arch Intern Med 1988;148: 2013-2016.

37. Winkleby MA, Kraemer HC, Ahn DK, Varady AN. Ethnic and socioeconomic differences in cardiovascular disease risk factors: findings for women from the Third National Health and Nutrition Examination Survey, 1988-1994. JAMA. 1998; 280:356-62.

38. Jacobsen BK, Thelle DS. Risk factors for coronary heart disease and level of education. The Tromso Heart Study. Am J Epidemiol 1988;127:923-932.

39. Lee JR, Paultre F, Mosca L. The association between educational level and risk of cardiovascular disease fatality among women with cardiovascular disease. Womens Health Issues 2005;15:80-88.

40. Feldman JJ, Makuc DM, Kleinman JC, Cornoni-Huntley J. National trends in educational differentials in mortality. Am J Epidemiol 1989;129:919-933.

41. Weissman JS, Stern R, Fielding SL, Epstein AM. Delayed access to health care: risk factors, reasons, and consequences. Ann Intern Med 1991;114:325-331.

42. Riegel B, Moser DK, Anker SD, Appel LJ, Dunbar SB, Grady KL, et al. State of the science: promoting self-care in persons with heart failure: a scientific statement from the American Heart Association. Circulation. 2009; 120:1141-63.

43. Brotman DJ, Golden SH, Wittstein IS. The cardiovascular toll of stress. Lancet 2007;370:1089-1100. 
44. Koelling TM, Johnson ML, Cody RJ, Aaronson KD. Discharge education improves clinical outcomes in patients with chronic heart failure. Circulation 2005;111:179-185.

45. Stromberg A. The crucial role of patient education in heart failure. Eur J Heart Fail 2005;7:363-369.

46. Ponikowski P, Voors AA, Anker SD, Bueno H, Cleland JGF, Coats AJS, et al. ESC Committee for Practice Guidelines. 2016 ESC Guidelines for the diagnosis and treatment of acute and chronic heart failure: the Task Force for the Diagnosis and Treatment of Acute and Chronic Heart Failure of the European Society of Cardiology. Developed in collaboration with the Heart Failure Association (HFA) of the ESC. Eur Heart J 2016;37:2129-2200.

47. Yancy CW, Jessup M, Bozkurt B, Butler J, Casey DE Jr, Colvin MM, et al. 2017 ACC/AHA/HFSA Focused Update of the 2013 ACCF/AHA Guideline for the Management of Heart Failure: A Report of the American College of Cardiology/American Heart Association Task Force on Clinical Practice Guidelines and the Heart Failure Society of America. J Card Fail. 2017;23:628-651.

48. Fabbri, M, Yost K, Finney Rutten LJ, Manemann SM, Boyd CM, Jensen D, et al. Health Literacy and Outcomes in Patients With Heart Failure: A Prospective Community Study Mayo Clin Proc. 2018;93:9-15.

49. Kirstensen SL, Kober L, Jhund PS, Solomon SD, Kjekshus J, McKelvie RS. et al. International geographic variation in event rates in trials of heart failure with preserved and reduced ejection fraction. Circulation 2015;131:43-53.

\section{FIGURE LEGENDS}

Figure 1 A-C. Kaplan-Meier plots for all-cause mortality of propensity matched patients with incident heart failure by income: $\mathbf{A}, \mathrm{HF}$ patients. $\mathbf{B}, \mathrm{HFrEF}$ patients. $\mathbf{C}, \mathrm{HFpEF}$ patients.

Figure 1 A-C suppl. Kaplan-Meier plots for cardiovascular mortality of propensity matched patients with incident heart failure by income: A, HF patients. B, HFrEF patients. C, HFpEF patients.

Figure 2 A\&B suppl. Estimated cumulative rate of hospitalization for HF worsening (per 100 patient years) (A) and of 30-day readmission for HF worsening, per 100 patients years (B ), by income in the matched groups. By three years, the cumulative number of HF hospitalizations (per 100 patient-years) is 8.8 for patients with $\mathrm{HF}$ in the low/middle income level, compared to 4.7 for those in the highest income level, a treatment difference of 4.1 per 100 patient-years (46.6\%) for HF patients in the highest income level, who avoid admission for HF. Beyond 3 years, these differences continue to increase for all studied groups.

Figure 3 A-C suppl. Relative risks (RR) and 95\% confidence intervals (CIs) for High vs. Low/Medium income in pre-specified subgroups.

\section{Hosted file}

Table 1 HFrEF AfterM.doc available at https://authorea.com/users/334958/articles/460903socioeconomic-status-and-prognosis-of-heart-failure-with-reduced-vs-preserved-ejectionfraction-a-propensity-matched-study-in-the-community

\section{Hosted file}

Table 2 HFpEF AfterM.doc available at https://authorea.com/users/334958/articles/460903socioeconomic-status-and-prognosis-of-heart-failure-with-reduced-vs-preserved-ejectionfraction-a-propensity-matched-study-in-the-community

\section{Hosted file}

Figure 1 Mort\&Income.pptx available at https://authorea.com/users/334958/articles/460903socioeconomic-status-and-prognosis-of-heart-failure-with-reduced-vs-preserved-ejectionfraction-a-propensity-matched-study-in-the-community

\section{Hosted file}


Table 3 Multivariate ITT.doc available at https://authorea.com/users/334958/articles/460903socioeconomic-status-and-prognosis-of-heart-failure-with-reduced-vs-preserved-ejectionfraction-a-propensity-matched-study-in-the-community 